Click here to view linked References

1

2

3

\section{Infer disease-associated microbial biomarkers based on metagenomic and} metatranscriptomic data

3 Zhaoqian Liu', Qi Wang ${ }^{1}$, Dongjun Chung ${ }^{2}$, Qin Ma², Jing Zhao,\#, Bingqiang Liü,\#

$4{ }^{1}$ School of Mathematics, Shandong University, Jinan, Shandong, 250200, China.

$5{ }^{2}$ Department of Biomedical Informatics, The Ohio State University, Columbus, $\mathrm{OH}, 43210$,

6 USA.

7 \#To whom correspondence should be addressed.

8

9 Email

10 Zhaoqian Liu --- Izqian8023@163.com;

11 Qi Wang --- wangqi1994_sdu@163.com;

12 Dongjun Chung --- dongjun.chung@osumc.edu;

13 Qin Ma --- qin.ma@osumc.edu;

14 Jing Zhao --- jing.zhao2@osumc.edu;

15 Bingqiang Liu --- bingqiang@sdu.edu.cn. 


\section{Abstract}

22 Unveiling disease-associated microbial biomarkers is crucial for disease diagnosis and

23 therapy. However, the heterogeneity, high-dimensionality, and large amounts of microbial 24 data bring tremendous challenges for fundamental characteristics discovery. We present

25 IDAM, a novel method for disease-associated biomarker inference from metagenomic and

26 metatranscriptomic data, without requiring prior metadata. It integrates gene context

27 conservation (uber-operon) and regulatory mechanism (gene co-expression patterns)

28 through a mathematical graph model. We applied IDAM to inflammatory bowel disease

29 associated matched metagenomic and metatranscriptomic datasets, which showed

30 superior performance in biomarker inference. IDAM is freely available

31 at https://github.com/OSU-BMBL/IDAM.

\section{Keywords}

33 Metagenome; Metatranscriptome; Microbial biomarkers; Gene regulation; Gene context 34 conservation; Unsupervised learning.

\section{Background}

36 Trillions of microbes colonize the human body and play critical roles in multiple

37 fundamental physiological processes, such as immune system development and dietary

38 energy harvest [1]. Moreover, massive evidence reveals that changes in microbial

39 composition and functions are intimately interwoven with multiple diseases ranging from

40 obesity to cancer and autism [1,2]. With the rapid development of sequencing

41 technologies, worldwide projects, such as the Human Microbiome Project [3], the

42 integrative Human Microbiome Project [4], and the Metagenomics of the Human Intestinal

43 Tract project [5], have generated vast amounts of microbial data for diverse diseases.

44 Thereafter, increasing efforts have been aimed at bringing these data to clinical insights 
45 and deepening the understanding of crucial mechanisms responsible for disease

46 progression and treatment. For example, recent studies have suggested that transferring

47 several microbes from a donor into a sick recipient can promote the recipient's recovery

48 and cause changes in the donor phenotype (e.g., increased adiposity) [6]. Furthermore,

49 some pathogenic organisms have been found closely related to disease states and can

50 be used as a potential marker of disease development [7, 8]. These findings hint at a

51 promising avenue for clinical application (disease diagnosis and therapy) via the use of

52 key microbial taxa or functions, i.e., microbial biomarkers.

53 Till now, a great deal of research has been explored for identifying microbial

54 biomarkers due to their critical roles in diseases. One of the classical paradigms is

55 identifying taxa and functions that can statistically significantly differentiate two or more

56 groups as biomarkers [9-11]. Additionally, given machine learning's success under

57 multiple scenarios, there are several attempts to adopt them in microbial biomarker

58 inference, such as random forest and deep feedforward networks $[12,13]$. However, these

59 approaches face several challenges.

60 First, most of them focus on $16 \mathrm{~S}$ ribosome RNA (16S rRNA) and metagenomic

61 (MG) data. 16S rRNA data suffer from a low taxonomic resolution and an absence of

62 functional information [14]. The taxonomic analysis alone may induce spurious biomarkers

63 since diverse microbial communities from different patients can perform remarkably similar

64 functional capabilities [14]. While MG data can provide information at all taxonomic levels

65 as well as potential functional information, researchers have found that: (i) A great

66 proportion of reads cannot be successfully mapped to existing reference genomes during

67 taxonomic identification, which leads to the potential loss of valuable information [15].

68 Intuitively, one may propose to use de novo assembled genomes as a complement.

69 However, the difficulties in metagenome assembly and binning generally cause incorrect

70 contigs, which substantially affect further taxonomic inferences [16]. (ii) The functional 
71 analyses from metagenomics are not equivalent to the true activities within the microbiome.

72 It has been observed that multiple metagenomically abundant genes in the human gut

73 microbiome were significantly down-regulated at the transcriptional level [17].

74 Second, existing methods are designed based on the strong assumption that the

75 data with sufficient sample size and accurate and detailed metadata information is

76 available to design groups or train models. However, the current metadata of a

77 considerable number of sequencing samples is incomplete, misleading, or not publicly

78 available [18], which may lead to these methods being infeasible or causing bias in

79 biomarker inference. Moreover, their intrinsic design in using known phenotype

80 information makes them incapable of revealing new subtypes or stages of diseases.

81 These challenges drive the need to develop an easy-to-use and effective method that

82 keeps up with current data for disease-associated microbial biomarker inference.

83 In this study, we propose a novel method to Infer ㅁisease-Associated Microbial

84 functional biomarkers (IDAM), which to a large extent surpasses these limitations

85 discussed above. Specifically, the data we used was matched MG and metatranscriptomic

86 (MT) data sequenced from microbial samples for assessing active functions within

87 communities. To make full use of available data, we focused on a community-level

88 functional analysis that goes beyond species level (by which the reads do not need to be

89 mapped to reference genomes), and provided a comprehensive overview of functions

90 performed by either individuals or different microbial assemblages. Furthermore, we

91 considered identifying functional biomarkers by detecting genes with specific co-

92 expression patterns across certain subsets of samples, without requiring prior metadata.

93 Undeniably, co-expression does not necessarily imply functional relevance [19]. Moreover,

94 one concern is that gene expression referred here is assessed by the reads from various

95 species (Fig. 1a), which represents an ensemble expression of multiple homologous

96 genes. Hence, we accept the fact that one cannot fully characterize the complex functional 
97 activities based solely on the ensemble co-expression, by which the genes with no

98 biological relevance may be clustered together (Fig. 1b). Fortunately, recent studies have

99 suggested that functionally associated gene sets are generally highly conserved during

100 evolution, i.e., uber-operon structures [20, 21]. This finding strongly motivated us to

101 integrate gene context conservation to study cross-species functional-related

102 mechanisms, thereby alleviating the risk of false positives or false negatives in biomarker

103 inference. Specifically, we used uber-operon structures as the evolutionary footprints of

104 cross-species functionally related genes. We believe that genes are more likely to be

105 functionally related if they have similar expression patterns and share the same uber-

106 operon during evolution. Therefore, we integrated both gene regulation and gene context

107 conservation to identify cross-species functional biomarkers in IDAM (Fig. 1c).

108 We applied IDAM to the published matched MG and MT datasets of inflammatory

109 bowel diseases (IBD) ( $n=813$; including two subtypes, ulcerative colitis (abbreviated as

110 UC) and Crohn's disease (abbreviated as CD)) [22]. It could identify 41 gene modules,

111 corresponding to 41 biomarkers. We validated the reliability of these biomarkers based on

112 known phenotypes and well-studied species/functions related to IBD reported previously.

113 Results suggested superior performance of IDAM in key biomarker inference compared

114 to popular tools, including both metadata-based (LEfSe [11]) and non-metadata-based

115 approaches (ICA [23], QUBIC [24], ISA [25], and FABIA [26]). Furthermore, IDAM

116 classified two IBD subtypes with high accuracy. Hence, we believe that IDAM can be a

117 highly advantageous method for biomarker inference based on MG and MT data. It can

118 potentially pave the way for understanding the role of the microbiome in human diseases

119 and improving disease diagnosis and treatment in clinical practice.

120

121

Please put Fig. 1 here. 
122 Fig. 1 The diagram of key ideas of IDAM. a. Sequencing data from four samples ( $p_{1}$ and

$123 p_{2}$ from healthy cohorts, and $p_{3}$ and $p_{4}$ from disease cohorts). Since metagenomic shotgun

124 sequencing does not isolate microorganisms, the reads from different species

125 (represented by black purple, black green, and black blue) were jumbled together. As a

126 result, we cannot distinguish them easily at the species level but we can observe the

127 expression of each gene only in a manner that reads from all species are merged together.

128 Note that the microbial community transcript number (MT data) can be corrected using the

129 underlying genomic copy number (MG data) in practice. Here, we simply showed the

130 number of transcriptomic reads as expressions. The colors of circles (grey, blue, and red)

131 indicate the expression level of genes within each sample. Here, the ground truth is

132 assumed that $g_{1}$ and $g_{2}$ are functionally related in healthy samples while $g_{4}$ and $g_{5}$ are

133 functionally related in disease samples. b. The co-expression modules. There were three

134 co-expression modules, which are defined as the gene and sample subsets with

135 coordinated expression patterns. Note that however they were not equivalent to the known

136 functional relationship. c. The functional modules by integrating uber-operon structures

137 and expression similarity. Each ellipse, including multiple genes, represents an uber-

138 operon. Here we search for modules consisting of genes with both co-expressed patterns

139 and shared uber-operons, which resulted in two identified modules. Therefore, we inferred

140 there were functional relationships between $g_{1}$ and $g_{2}$, as well as $g_{4}$ and $g_{5}$, which were

141 consistent with the ground truth.

142

143 Results

144 IDAM: a metagenomic and metatranscriptomic analysis framework for disease145 associated microbial biomarker inference 
146 We first present the problem formulation of this article and then provide an algorithmic 147 overview of IDAM.

\section{Problem formulation}

149 The problem of biomarker inference can be mathematically formulated as an optimization 150 problem. For matched MG and MT data, a gene-sample expression matrix $A=\left(a_{i j}\right)_{m \times n}$ 151 can be constructed, which includes $m$ genes $\left(g_{1}, g_{2}, \ldots, g_{\mathrm{m}}\right)$ and $n$ samples $\left(p_{1}, p_{2}, \ldots, p_{n}\right)$. $152 a_{i j}(1 \leq i \leq \mathrm{m}, 1 \leq j \leq n)$ represents the expression of gene $g_{\mathrm{i}}$ within sample $p_{\mathrm{j}}$, which is 153 calculated by normalizing RNA abundance (MT data) against DNA abundance (MG data) 154 of $g_{\mathrm{i}}$ within sample $j$. Based on the matrix, the co-expression patterns present in gene and 155 sample subsets can be detected to identify local low-rank submatrices [27]. Given the 156 constraints of gene context conservation, we here integrate the submatrices with a graph 157 model to maximize the number of genes connecting to a single uber-operon. Specifically, 158 define a bipartite graph $G=\left(V_{1}, V_{2}, E\right)$, where each node $u \in V_{1}$ represents an uber159 operon. Each node $v \in V_{2}$ represents a gene in $A$, and the edge $e \in E$ connecting a 160 node $u \in V_{1}$ to a node $v \in V_{2}$ exists if the gene $v$ belongs to uber-operon $u$ (Methods). 161 Using this graph and matrix $A$, the biomarker inference problem can be formulated as 162 identifying a set of local low-rank submatrices within $A$ that maximizes the number of 163 connected components between the corresponding gene sets and $V_{1}$ on $G$. Note that here 164 we maximize the number of connected components between gene subsets and $V_{1}$ to 165 minimize the heterogeneity of genes for each uber-operon, thereby ensuring the genes 166 within each identified submatrix as functionally related as possible. Based on the identified 167 submatrices within $A$ (each of which contains a subset of genes and samples), the gene 168 sets can be regarded as biomarkers of the shared phenotypes of the sample sets. 169 However, this problem is theoretically intractable (NP-hard, Additional file 1: Section 1). 
170 Hence, instead of trying to solve it directly, we develop a heuristic algorithm, IDAM, as an

171 approximate optimal solution.

\section{Overview of IDAM}

174 The main goal of IDAM is to identify disease-associated microbial biomarkers (Fig. 2a).

175 Firstly, the community-level expression within each sample is assessed by HUMAnN2 176 based on the matched MG and MT data [28]. This step provides a gene-sample 177 expression matrix, each entry of which represents the expression of a gene within a 178 sample. Secondly, IDAM combines expression similarity and gene distribution within uber179 operons to measure the likelihood that each pair of genes is involved in the same function. 180 This step provides a list based on combined assessment for module initialization (Fig. 2b). 181 Thirdly, the gene pairs in the obtained list are used as seeds one by one, and each seed 182 is expanded by iteratively adding other functionally related genes to generate modules 183 until the new module starts to become smaller than the previously identified ones (Fig. 2c). 184 Finally, the gene modules consisting of gene sets and corresponding sample sets will be 185 considered as the final output of the algorithm. Here we will regard gene sets as functional 186 biomarkers of the most common phenotype or state of corresponding samples. Details 187 are available in the "Methods" section.

189 Fig. 2 The workflow of IDAM. a. The flow chart. We consider matched MG and MT data 190 of multiple samples as input, from which we obtain an expression matrix. By module 191 initialization and expansion, the modules will generate as output. b. Details of module 192 initialization. For each gene pair, we assess both expression similarity and context 193 conservation for a combined score. Let us use $g_{3} g_{4}$ as an example. Based on known uber194 operon structures, the two genes belong to $u_{2}$. We assign a reward $U$ for $g_{3} g_{4}$ as context 
195 conservation measurement. Expression similarity is assessed according to the number of

196 samples under which the rows are identical, and we get a score $S$ (Methods). Finally, $U$

197 and $S$ are combined via a function $C$ ( $w$ is a tuning parameter), obtaining a score $C_{34}$. All

198 gene pairs are sorted in a decreasing order based on combined scores. c. Details of

199 module expansion. We use gene pairs in the list as initial modules and expand them

200 gradually. Specifically, we iteratively add a new gene with the highest combined score into

201 a module until the updated module does not get bigger. Let us use $g_{3} g_{4}$ as an example.

202 First, we add three genes $\left(g_{1}, g_{2}\right.$, and $\left.g_{5}\right)$ into $g_{3} g_{4}$, respectively, forming three new

203 modules (denoted as $g_{3} g_{4} g_{1}, g_{3} g_{4} g_{2}$, and $g_{3} g_{4} g_{5}$ ). Then, combined score of each module

204 is assessed (Methods). Since the module $g_{3} g_{4} g_{5}$ is of the highest score, we replace the

205 previous with this one. Next, iteratively add a new gene $\left(g_{1}\right.$ and $\left.g_{2}\right)$ to the current module

206 and implement the same processes. After comparing the combined scores, we find

$207 g_{3} g_{4} g_{5} g_{2}$ is of the highest score, but the module is of size $4 \times 2$, which is smaller than $g_{3} g_{4} g_{5}$

$208(3 \times 3)$. Therefore, we stop here and the module $g_{3} g_{4} g_{5}$ will be provided as the final output.

210 The biomarkers inferred by IDAM well distinguished different phenotypes and 211 enabled the discovery of disease subtypes

212 To assess the usefulness of biomarkers identified by IDAM, we assessed the phenotype 213 classification performance using identified biomarkers. We applied IDAM to the publicly 214 available dataset of patients with IBD and controls (referred to as Non-IBD) (Methods, all 215 following analyses are based on these datasets). By assessing the community-level 216 expression within each sample, we obtained a matrix consisting of the expression of 217941,785 genes across 813 samples. Based on this, IDAM found 41 gene modules with 218 genes spanning from 2,340 to 41, totally covering 19,327 genes (Additional file 2: Table 219 S1). The gene set contained in each module was regarded as a biomarker. That is, there 
220 were 41 identified biomarkers. Ideally, samples with the same biomarker should have

221 highly consistent phenotypes. We used Z-test to evaluate the phenotype consistency of 222 samples in each module (Methods). Results showed that 32 biomarker-associated sample 223 sets were consistently enriched for a particular phenotype (19 enriched for IBD and 13 for 224 Non-IBD, Fig. 3a, Additional file 2: Table S2). On the other hand, we recognized that 225 microbial communities can be influenced by multiple factors such as age, race, and diet.

226 These confounding factors can lead to high inter-individual heterogeneity, make disease227 associated microbial characteristics less clear, and increase the risk of false positives or 228 negatives in biomarker inference. Hence, we further assessed whether the identified 229 biomarkers from IDAM had reliable associations with phenotypes, by applying a 230 propensity score-based matching for each biomarker to confirm its association with 231 phenotypes. Specifically, we constructed paired samples by matching the propensity 232 score estimated from other covariates, such as age, gender, and comorbidities (Methods). 233 Wilcoxon signed-rank test based on the paired samples indicated that $82.93 \%$ (34 of 41 ) 234 biomarkers were still statistically significantly related to a specific phenotype (with 235 Benjamini-Hochberg-adjusted $p$-value < 0.05, Fig. 3b, Additional file 2: Table S3), 236 demonstrating high reliability of identified biomarkers from IDAM.

237 Additionally, we observed statistically significant enrichment of the disease 238 subtypes within each module. A noteworthy fact was that the identified biomarkers could 239 recognize the subtypes of IBD, in which 11 correspond to $\mathrm{UC}$ and eight correspond to CD 240 (Fig. 3c). We here gave an example of three modules to show the phenotype specificity 241 (Fig. 3d), namely Module 1, Module 17, and Module 30, each of which is enriched for a 242 particular phenotype. Limited by space, we showed the expression of the top 3\% genes 243 of each module (17/618, 19/689, and 14/521 for Modules 1, 17, and 30, respectively). We 244 can see that IDAM detected the classical checker-board substructures from the original 245 expression data. Specifically, Biomarker 1 is specific to Non-IBD while Biomarker 17 and 
24630 are specific to $U C$ and $C D$, respectively, suggesting the capability of IDAM to uncover

247 biomarkers of disease subtypes. We further carried out functional enrichment analysis for 248 inferred biomarkers from IDAM (Methods). Results showed that these biomarkers were 249 associated with 168 pathways (Additional file 2: Table S4), of which 27 were also identified 250 as phenotype-differential pathways based on pathways abundance analysis of HUMAnN2 251 (Methods, Additional file 2: Table S4). For example, there was a significant enrichment of 252 short-chain fatty acids metabolism (e.g., butyrate metabolism) in Non-IBD-associated 253 biomarkers. L-methionine biosynthesis, superpathway of L-citrulline metabolism, and 254 superpathway of L-lysine, L-threonine, and L-methionine biosynthesis were enriched in 255 Non-IBD-associated biomarkers, while preQ biosynthesis was enriched in IBD. 256 Additionally, the superpathway of lipopolysaccharide biosynthesis was enriched in CD257 associated biomarkers while methylphosphonate degradation was enriched in UC258 associated biomarkers. These findings are paralleling with previous observations [29-32], 259 indicating that the biomarkers from IDAM were biologically informative in distinguishing 260 phenotypes.

261 We compared IDAM with several popular biclustering-based and decomposition262 based approaches, including ICA [23], QUBIC [24], ISA [25], and FABIA [26] (Methods). 263 These methods have not been applied to microbial data for biomarker inference but 264 intuitively they can reveal functional characteristics as biomarkers under different 265 phenotypes without the need for prior metadata. Results suggested that there were 34, 26630 , and 13 biomarkers identified by QUBIC, ICA, and FABIA, respectively, while no 267 biomarker was detected by ISA. We further evaluated the phenotype consistency of 268 biomarker-associated sample sets. While 78.05\% (32/41) sample sets from IDAM were 269 with a consistent phenotype, only $61.76 \%(21 / 34), 33.33 \%(10 / 30)$, and $30.77 \%(4 / 13)$ for 270 QUBIC, ICA, and FABIA, respectively (Fig. 3e). This revealed that the inferred biomarkers 271 from IDAM can best cluster the samples with the same phenotypes together compared 
272 with others, demonstrating the high effectiveness in distinguishing phenotypes. Indeed,

273 this finding was not surprising since IDAM took both gene expression and gene context

274 conservation into consideration, thereby identifying more biologically meaningful

275 biomarkers, while others intrinsically used only gene expression. Detailed analyses can

276 be found in the following subsection "The integration of gene context conservation

277 substantially improves the performance of biomarker inference". Finally, we compared the

278 running time of these methods (Fig. 3f). IDAM spent 7 hours, QUBIC and FABIA took 16

279 and 22 hours, respectively, while ISA was terminated after 24 hours without results. ICA

280 costs 8 minutes; however, the application power of ICA is limited by the relatively low

281 accuracy (only one-third of the biomarkers can distinguish phenotypes).

282 Collectively, the biomarkers identified by IDAM performed best in distinguishing 283 phenotypes and can provide hints for new disease subtypes or states in clinical application.

Please put Fig. 3 here.

286 Fig. 3 The performance of inferred biomarkers by IDAM in distinguishing phenotypes. a. 287 The Z-scores of Non-IBD, UC, and CD. They were respectively shown as pink, green, and 288 purple, and each column represents a biomarker. The yellow horizontal line means the 289 critical value of 1.645 . The color of circles above this line means that the corresponding 290 phenotype exhibited strong enrichment within the biomarker-associated sample sets. b.

291 The adjusted $p$-values of each biomarker with respect to different phenotypes. $p$-values 292 were obtained using the Wilcoxon signed-rank test based on propensity score matching, 293 followed by multiple testing adjustments using the Benjamini-Hochberg procedure. Each 294 row represents a biomarker, and the color means adjusted $p$-value. * indicates adjusted 295 p-value < 0.05. c. The number of biomarkers identified for each phenotype. A total of 19 296 biomarkers correspond to IBD (in which 11 to UC and 8 to CD) and 13 correspond to Non- 
297 IBD. d. An example of gene modules associated with phenotypes, where Module 1, 17,

298 and 30 are shown. The colors of each cell in the heatmap indicate the log2 value of the

299 expression. e. The percent of biomarkers associated with samples enriched in certain

300 phenotypes, identified by IDAM and competing algorithms. NA means no results from ISA.

301 f. The running time of IDAM and competing algorithms.

302

303

Species-level analysis suggested the biomarkers inferred by IDAM were biologically associated with phenotypes

305 We implemented species-level analysis for biomarkers from IDAM. Since differences in 306 community-level functions ultimately imply species-level functional differences, we traced 307 genes within each biomarker back to specific species (Methods). We found that these 308 genes were from 111 species, which were dominated by Bacteroidetes and Firmicutes, 309 followed by Proteobacteria at the phylum level (Fig. 4a). This observation was consistent 310 with previous research about IBD-associated species [32]. We further classified the 311 species according to biomarker-associated phenotypes (Methods) and obtained 37, 64, 312 and 13 species associated with Non-IBD, UC, and CD, respectively (Additional file 2: 313 Table S5). Three of them were overlapped between UC and CD, including Flavonifractor 314 plautii, Bacteroides vulgatus, and Bacteroides fragilis. We collected IBD-related species 315 (34 for Non-IBD, 32 for UC, and 90 for CD) from Peryton (a database of microbe-disease 316 associations) and previous taxonomic studies of IBD [33-36] (Additional file 2: Table S6). 317 Among them, a total of 29 species were captured by IDAM (Fig. 4a), including 17 for Non318 IBD, 6 for UC, and 9 for CD (shown in red in Additional file 2: Table S6). An interesting 319 finding was that the three overlapped species from IDAM have been reported associated 320 with both UC and CD [33-35], suggesting the rationality of IDAM results. 
We compared IDAM with QUBIC [24], ICA [23], FABIA [26], and LEfSe [11]

322 (Methods). Note that ICA, QUBIC, and FABIA are not dependent on metadata while LEfSe

323 identifies differential abundant species as biomarkers based on prior phenotype

324 information. Detailed results are shown in Fig. 4b. Not surprisingly, the average

325 consistency with collected species across three phenotypes was highest for IDAM

326 (41.52\%), followed by QUBIC (19.82\%), LEfSe (18.38\%), ICA (17.62\%), and FABIA

327 (4.74\%) (Methods, Fig. 4c). Collectively, the species-level analysis showed the inferred

328 biomarkers from IDAM were biologically associated with phenotypes.

Please put Fig. 4 here.

331 Fig. 4 The species-level analysis of identified biomarkers. a. The phylogenetic tree of the 332 species associated with biomarkers identified by IDAM. The colored dots indicated 333 different species that have been validated by previous studies. $\mathbf{b}$. The results of species334 level analysis by different methods. The numbers in the header (34, 32, and 90) represent 335 the number of collected species associated with the three phenotypes, respectively. Each 336 cell in the table is represented in the form of $X / Y(Z)$, where $Y$ represents the number of 337 species associated with a particular phenotype based on different methods, among which 338 consistent with the collected species are represented as $X$, and $Z$ indicates the 339 consistency index of each method associated with phenotypes. c. The mean consistency 340 across three phenotypes was used as the average consistency index for each method. 341 The greater value means that identified biomarkers-associated species are more reliable.

343 The integration of gene context conservation substantially improved the 344 performance of biomarker inference 
345 We investigated the contribution of gene context conservation, given our underlying 346 hypothesis that genes with similar expression patterns and high context conservation 347 during evolution are more likely to be functionally related. To evaluate this, we sought to 348 identify biomarkers without using gene context conservation in IDAM (referred to as IDAM 349 without gene context conservation). Results showed that biomarkers identified by this 350 version are significantly less capable of distinguishing different phenotypes than those 351 from IDAM (Fig. 5a). Specifically, IDAM without gene context conservation detected 34 352 gene modules from the expression matrix, of which only $61.76 \%(21 / 34)$ sample subsets 353 were of consistent phenotypes. In contrast, in the case of IDAM, 78.05\% (32/41) sample 354 subsets were of consistent phenotypes. The pathway analysis suggested that $67.65 \%$ 355 (23/34) biomarkers from IDAM without gene context conservation were significantly 356 enriched in MetaCyc functional categories, which is significantly lower than $82.93 \%(34 / 41)$ 357 of IDAM. These strongly indicated that the integration of uber-operon structures 358 contributed to identifying informative biomarkers.

359 We further explored underlying reasons for the superior performance of integrating 360 gene context conservation. For this purpose, for each biomarker (from either IDAM or 361 IDAM without gene context conservation), we evaluated the distribution of contained 362 genes in the uber-operons. Overall, we found that the genes within biomarkers that cannot 363 significantly enrich certain functions tended to be spread out among more uber-operons, 364 compared with those within biomarkers that enrich. This finding supports our hypothesis 365 that functionally related gene sets are of high context conservation. As an example, we 366 further investigated Module 32 from IDAM without gene context conservation (abbreviated 367 as 'Module 32' hereinafter, Fig. 5b). Since the 42 genes within the module were of 368 coordinated expression within the 80 samples, they would be clustered together when we 369 merely considered co-expression. However, these genes were not significantly 370 functionally related and were from six uber-operons (Fig. 5b \& 5c). They cannot reflect the 
371 unique functional characteristics of a particular phenotype. Not surprisingly, the 80

372 samples within Module 32 were evenly scattered over different phenotypes (22 with Non-

373 IBD, 22 with UC, and 36 with CD). When we took gene context conservation into

374 consideration (i.e., IDAM), a new module occurred (e.g., Module 31 from IDAM,

375 abbreviated as 'Module 31' hereinafter). It consists of 85 genes and 34 samples, and there

376 was considerable overlap with Module 32 (Fig. 5b). This module was dominated by an

377 uber-operon that closely associates with purine metabolism [21], and the significantly

378 enriched functions were shown in Fig. 5c. Previous studies have shown that the imbalance

379 between biosynthesis and degradation of purine metabolism could produce excessive uric

380 acid in the gut, leading to the occurrence of IBD [37]. As expected, the 34 samples were

381 enriched in phenotype CD. This demonstrated the strong power of gene context

382 conservation in clustering genes with biological relevance together, which improves the

383 ability of IDAM for biomarker inference.

384

385

Please put Fig. 5 here.

386 Fig. 5 The analysis of the contribution of gene context conservation. a. Comparison 387 between IDAM and IDAM without gene context conservation. The red bars mean the 388 results from IDAM, while the grey bars mean results from IDAM without gene context 389 conservation. b. A specific case of identified modules. Each row represented a gene and 390 each column represented a sample (totally 101 genes and 80 samples). The grey 391 rectangle highlights Module 32 identified by IDAM without gene context conservation, and 392 the red highlights Module 31 identified by IDAM. The colored bar on the top represented 393 different phenotypes of corresponding samples. Circle colors on the right represented the 394 genes that belong to the same uber-operons. c. The pathways enriched for Module 32 395 and Module 31. The grey ones mean the pathways enriched for Module 32 while red 396 means those enriched for Module 31. The dotted vertical lines mean the critical value with 
397 a $p$-value of 0.05 . The pathways located outside of the dotted lines correspond to 398 significantly enriched pathways.

399

400 Discussion

401 Although many studies have focused on the microbial biomarker inference of diseases, it 402 is still a fundamental challenge to explore critical characteristics in large-scale and highly 403 heterogeneous microbial data. We developed a new methodology, IDAM, for matched MG 404 and MT data to identify disease-associated biomarkers. IDAM is innovative in the sense 405 that, unlike the previous microbial biomarker analysis methods, it integrates gene context 406 conservation and regulation information by leveraging a multi-omic view of microbial 407 communities for functional characteristics inference of diseases. Furthermore, IDAM does 408 not require any prior knowledge about samples, which alleviates bias from misleading data 409 noise and allows us to reveal novel disease subtypes or states. We tested IDAM for the 410 purpose of IBD biomarker inference and found that it remarkably outperformed existing 411 methods. We discovered 41 significant microbial biomarkers associated with IBD, where 412 some of their functions are consistent with what has been reported previously. In addition, 413 we evaluated the gain of using gene context conservation information in IDAM and found 414 that ignoring this information can lead to significantly decreased biological relevance 415 between genes within a module. This demonstrated that uber-operon structures played a 416 crucial role in functional indication. Notably, although we use IBD to illustrate and evaluate 417 IDAM, IDAM can contribute to the understanding of other diseases.

418 Admittedly, IDAM is still not free of limitations. First, IDAM identifies biomarkers at 419 the community level, which needs further taxonomic analysis to improve their utility as 420 clinical biomarkers. This limitation can be somewhat addressed by tracing the community421 level functions back to the taxonomic level using HUMAnN2. However, there are some 
422 biomarkers that attribute to unknown species (labeled as unclassified species in

423 HUMAnN2). Therefore, cautious inference is needed in interpretation of these biomarkers

424 since we cannot confidently say whether they are actually false biomarkers, or instead

425 simply correspond to species that have not been well studied. However, we believe that

426 this issue will be alleviated as more microbes are discovered and explored. Given this, we

427 plan to develop a three-dimensional analysis approach in the future, which takes both

428 microbial species and genes (pathways) of multiple samples into consideration for

429 biomarker inference. In this way, the specific modules with diseases consisting of function

430 (genes/pathways) and species will be detected, simultaneously providing functional and

431 taxonomic insights into diseases. Second, IDAM requires relatively large-scale data. Since

432 IDAM uses expression similarity to assess the relevance of gene regulation, a larger

433 sample size will result in more reliable measurement from data. In practice, the sample

434 pool can be an approach that addresses this issue and guarantee the practicability of

435 IDAM. Finally, the provided biomarkers of IDAM only provided associations between

436 microbiomes and diseases. Therefore, rigorous experiments are required to establish

437 causality between the identified biomarkers and the disease of interest, and establish

438 clinical prevalence and utility.

440 Conclusions

441 IDAM provides a highly effective approach to identify disease-associated microbial 442 biomarkers based on both gene regulation and context conservation during evolution, 443 based on matched MG and MT data. The identified biomarkers of diseases can be useful 444 in understanding disease pathogenesis and providing guidance for future development of 445 disease diagnosis and treatment. 


\section{Methods}

\section{Datasets}

449 We downloaded all matched quality-controlled MG and MT data, as well as the 450 corresponding HUMAnN2 results and metadata of samples at the IBDMDB website in 451 March 2020 (https://ibdmdb.org) [22]. In total, there are 198 samples from Non-IBD 452 subjects and 615 samples from patients with IBD (consisting of 381 UC samples and 234 453 CD samples). Uber-operon structures were from the paper of Che et al. [21].

454 IDAM: a framework of identifying disease-associated microbial biomarkers based 455 on metagenomic and metatranscriptomic data

456 IDAM is a heuristic algorithm for disease-associated microbial biomarker inference. It is 457 based on a greedy idea to gather genes with similar expression patterns and high context 458 conservation as functional biomarkers. IDAM consists of three steps.

\section{Step 1: Extracting community-level gene expression of samples.}

460 The input data is quality-controlled (quality- and length-filtered, and screened for residual 461 host DNA) matched MG and MT sequencing datasets (fasta, fasta.gz, fastq, or fastq.gz).

462 The datasets of each sample are firstly processed using HUMAnN2 for gene relative 463 expression within communities [28]. In this study, we directly used the HUMAnN2 results 464 of the datasets from IBDMDB. Then, we extract the community-level gene expression of 465 each sample to construct a gene-sample expression matrix $A_{m \times n}$, in which each row 466 represents a gene (totally $m$ genes indicating by UniRef90 gene family [38] and forming a 467 gene set $X$ ), and each column represents a sample (totally $n$ samples). 
470 We assess context conservation and expression similarity for each gene pair and sort

471 them as a list for module initialization.

\section{$472 \quad 2.1$ Gene context conservation}

473 Gene context conservation is assessed based on gene distribution in uber-operons. Since

474 the genes within $X$ referred here are indicated as gene families from UniRef90 (a non-

475 redundant protein sequence database) [38], we align the gene family sequences with

476 genes in uber-operons to determine which uber-operons these gene families belong to.

477 Specifically, the sequences of genes in all uber-operons are collected as a custom 478 database. For each gene family $g_{\mathrm{i}}$ within $X(1 \leq i \leq m)$, we extract the corresponding 479 sequence from the Uniprot database [38] and align it with the custom database using Blast 480 [39]. We define a gene-to-uber-operon mapping function $\varphi: D \rightarrow H$, where $D$ consists of 481 all subsets of $X, Y$ is the set of all uber-operons, and $H$ consists of all subsets of $Y$. We 482 assume that a gene family $g_{\mathrm{i}}(1 \leq i \leq m)$ belongs to an uber-operon $u$ if the $E$-value of its 483 alignment with one of the uber-operon's sequences is less than 0.001 . This is denoted by $484 \varphi\left(G_{i}\right)=\{u\}$, where $G_{\mathrm{i}}$ is the set consisting of single gene $g_{\text {i. }}$. If multiple sequences from 485 different uber-operons satisfy the $E$-value less than 0.001 , we will choose the uber-operon 486 corresponding to the smallest $E$-value. By contrast, if no sequence can satisfy the $E$-value 487 less than 0.001 , we set $\varphi\left(G_{i}\right)=\{\varnothing\}$. In this way, we construct a map between uber-operon 488 and a gene.

489 We further define the relationship between uber-operons and gene sets with 490 multiple genes. Suppose a gene set $Q$ consists of $b$ genes $\left\{g_{1}, g_{2}, \cdots, g_{b}\right\}(1<b \leq m)$, 491 we assume $\varphi(Q)=\varphi\left(\left\{g_{1}\right\} \cup\left\{g_{2}\right\} \cup \cdots \cup\left\{g_{b}\right\}\right)=\varphi\left(G_{1} \cup G_{2} \cup \cdots \cup G_{b}\right)=\varphi\left(G_{1}\right) \cup \varphi\left(G_{2}\right) \cup$ $492 \cdots \cup \varphi\left(G_{b}\right)$, where $G_{\mathrm{i}}$ represents the set consisting of single gene $g_{\mathrm{i}}(i=1,2, \ldots, \mathrm{b})$. For 493 two gene sets $I$ and $J$, we set a reward $U(I, \mathcal{J})$ as gene context conservation measurement 494 based on relative positions of their contained genes in uber-operons: 


$$
U(I, J)=\left\{\begin{array}{rc}
1, & \varphi(I) \subseteq \varphi(J) \text { and } \varphi(I) \neq\{\varnothing\} \\
-1, \quad & \varphi(I)=\{\varnothing\} \text { and } \varphi(J) \neq\{\varnothing\} \\
-1, \quad & \varphi(I) \neq\{\varnothing\} \text { and } \varphi(J)=\{\varnothing\} \\
-2, & \varphi(I) \neq \varphi(J) \neq\{\varnothing\} \\
0, & \text { otherwise. }
\end{array}\right.
$$

496 Based on this, we calculate $U\left(G_{i}, G_{j}\right)$ as gene context conservation of each pair of genes

$497 \quad g_{\mathrm{i}}$ and $g_{\mathrm{j}}(1 \leq \mathrm{i} \leq \mathrm{j} \leq m)$.

\section{$498 \quad 2.2$ Expression similarity of genes}

499 Expression similarity is assessed based on the vector composed of expression values of 500 each gene within all samples. Note that here we need to measure expression similarity 501 not only between two genes, but also across multiple genes, while expanding modules in

502 Step 3. This cannot be achieved by existing similarity measurements (e.g., Pearson 503 correlation coefficient) since they are generally used for paired data points. To address 504 this, the continuous expression value of each gene is first discretized to an integer 505 representation by qualitative representation [24] (Additional file 1: Section 2). Based on 506 this, we measure the expression similarity $S\left(G_{i}, G_{j}\right)$ of gene pair $\left(g_{i}, g_{j}\right)$ as the number of 507 samples under which the corresponding integers along the rows of the two genes are 508 identical (or identical integers but with opposite signs).

$510 \quad 2.3$ The integration of gene context conservation and expression similarity

511 A combined score $C\left(G_{i}, G_{\mathrm{j}}\right)$ integrating gene context conservation and expression 512 similarity of $g_{\mathrm{i}}$ and $g_{\mathrm{j}}$ is obtained via the following function,

$$
C\left(G_{i}, G_{j}\right)=\left(1+w \times U\left(G_{i}, G_{j}\right)\right) \times S\left(G_{i}, G_{j}\right)
$$

514 where $w$ is a tuning parameter that can be set by users. $w$ ranges between 0 and 1 , and 515 a larger value of $w$ indicates that gene context conservation has a stronger impact on 516 estimating functional relationships. Here we used 0.1 as the default value (Additional file 
517 1: Section 3 and Additional file 2: Table S7). Finally, the gene pairs are sorted in a 518 descending order for the combined scores, generating a seed list $L$.

\section{Step 3: Generating modules consisting of gene sets and corresponding sample sets}

520 Since the gene set $X$ is a pan-genome of all microorganisms from multiple samples, it

521 consists of a large number of genes. To improve computational efficiency, we partition the 522 rows within the matrix equally into $t$ subsets [40,41], where $t$ is determined by the 523 stochastic model $[40,41]$. Then, we use gene pairs within each subset instead of all gene 524 pairs in $X$, forming the seed list $L$, which can greatly decrease the number of seeds and 525 reduce computation burden.

526 A submatrix is regarded as biologically relevant if it does not occur randomly in 527 matrix $A_{m \times n}$. Since the output submatrices from our algorithm are biologically relevant 528 and generated by a gene pair (see Step 3.1), we need to make sure at least one gene pair 529 within each meaningful submatrix can exist as a seed to keep all biologically relevant 530 submatrices detectable. This means we can at most divide the rows of matrix $A$ into $k-1$ 531 subsets (i.e., $t=k-1$ ), where $k$ represents the number of genes of the biologically relevant 532 submatrix with the fewest genes (referred to with the smallest row size). We statistically 533 assess the smallest row size of the submatrix that is unlikely to occur by chance. Note 534 that, the submatrices we target are of rank one mathematically since the corresponding 535 genes are with co-expressed patterns. Suppose a submatrix with rank one is of size $k \times$ $536 s$, its probability of random occurrence in $A_{m \times n}$ is calculated as follows [40, 41]: $P_{k s}=n \times(n-1) \times \cdots \times(n-s+1) \times\left(\begin{array}{c}m \\ k\end{array}\right) \times\left(\frac{2}{s !}\right)^{k} \times\left(1-\frac{2}{s !}\right)^{m-k}$

538 We here think the submatrix is biologically relevant if $P_{k s}<0.05$. When users have prior 539 knowledge of the largest column size $s$, we can calculate $k$ based on the formula 540 mentioned above. By this, the smallest row size of biologically relevant submatrices can 
541 be determined. Based on the above, users can set the number of subsets $(t=k-1)$. In this

542 paper, the default value of $t$ is set to 10 . That is, the matrix will be divided into 10 subsets,

543 which can greatly reduce the computation burden and keep biologically relevant 544 submatrices detectable to a large extent.

\section{$546 \quad 3.1$ Initialization}

547 We start with the first gene pair in $L$ that satisfies i) at least one gene of these two has not

548 been included in previous modules, or ii) these two genes are respectively included within 549 different previous modules, and there is no overlap between the gene sets of the two 550 modules. We aggregate samples under which the integers of the two genes are identical 551 (represented as set $P$ ), forming current module $M=\{Q, P\}$, where $Q$ represents the gene 552 set consisting of the paired genes. Then, the gene pair is removed from $L$.

\section{$553 \quad 3.2$ Expansion}

554 We expand $M$ by adding a new gene (if any) that has the highest combined score with $M$, 555 giving rise to the updated module $M^{\prime}=\left\{Q^{\prime}, P\right\}$. The calculation of the combined score 556 between a gene (represented as $g_{z}(1 \leq z \leq m)$ ) and $Q$, namely $C\left(G_{z}, Q\right)$, is similar to 557 that of two genes $g_{\mathrm{i}}$ and $g_{\mathrm{j}}$. Specifically, we first assess the context conservation between $558 g_{z}$ and the gene set $Q$ of module $M$, i.e., $U\left(G_{z}, Q\right)$. Then, we measure the expression 559 similarity of $g_{z}$ and $Q, S\left(G_{z}, Q\right)$, as the number of samples within $P$ under which the 560 corresponding integers along the rows are identical (or identical integers but with opposite 561 signs). Based on these, a combined score $C\left(G_{z}, Q\right)$ can be obtained by replacing $U\left(G_{i}, G_{j}\right)$ 562 and $S\left(G_{i}, G_{\mathrm{j}}\right)$ in the calculation of $C\left(G_{\mathrm{i}}, G_{\mathrm{j}}\right)$ with $U\left(G_{\mathrm{z}}, Q\right)$ and $S\left(G_{\mathrm{z}}, Q\right)$, respectively. If $563 \min \left\{\left|Q^{\prime}\right|,\left|P^{\prime}\right|\right\}>\min \{|Q|,|P|\}$, we will set $M=M^{\prime}$ and repeat Step 3.2. Otherwise, go to Step 5643.3.

$565 \quad 3.3$ Filter and output 
566 If the proportion of overlapped genes between $Q$ and the gene sets of previously identified

567 modules is less than a threshold $f$, module $M$ will be provided as the final output, and it is

568 ignored otherwise. In this paper, the default $f$ is set to 0.1 for more separate modules. Go

569 to Step 3.1 until the list $L$ is empty.

570

571 Evaluating phenotype consistency of samples within one module

572 Z-test was used to assess whether samples with the same biomarker were of highly

573 consistent phenotypes. Specifically, we extracted the phenotypes of all samples from

574 metadata as background and the corresponding phenotypes of samples in each gene

575 module as test sets. For each phenotype (Non-IBD, UC, and CD) and each test set, we

576 counted the phenotype occurrence in the test set and background, respectively. We

577 evaluated the significance of each phenotype using a right-tailed Z-test with the null

578 hypothesis that the observed frequency in the test set was less than or equal to the

579 occurrence probability within the background. The threshold of significant level 0.05 was

580 used with a critical Z-value of 1.645 . If Z-score was greater than 1.645 ( $p$-value $>0.05$ ),

581 the null hypothesis would be rejected, meaning the phenotype significantly occurred within

582 the test set. In this case, we would consider the samples within the gene module was of a

583 highly consistent phenotype.

585 The propensity score matching method

586 Our aim was to assess whether the association between identified biomarkers and 587 disease phenotypes was reliable. For each biomarker, we firstly identified underlying 588 confounding variables that affected its occurrence. Here, we incorporated diet, age, race, 589 health conditions of other diseases, as well as the other biomarkers and calculated a 
590 propensity score for each sample (using R package Matchlt 4.2.0). Then, the paired 591 samples were constructed by matching with the optimal score (by setting the parameter 592 method = "optimal"). Finally, Wilcoxon signed-rank test was used to measure the 593 significance of each biomarker that associates with different phenotypes, and the $p$-value 594 was adjusted by the Benjamini-Hochberg procedure, with an adjusted $p$-value $<0.05$ as 595 significantly associated.

596

597 Functional analysis of biomarkers

598 We performed an analysis of the enriched functions of each biomarker. All UniRef90 599 identifiers were firstly mapped to MetaCyc reactions [28, 42]. The reactions were further 600 associated with MetaCyc pathways [28, 42], which enabled a transitive association 601 between UniRef90 gene families and MetaCyc pathways. We defined a pathway that was 602 satisfied when the reactions within the pathway significantly occurred via the 603 hypergeometric test,

604

$$
P\left(t_{i j}\right)=\frac{\left(\begin{array}{c}
n_{j} \\
t
\end{array}\right)\left(\begin{array}{l}
N-n_{j} \\
m_{i}-t
\end{array}\right)}{\left(\begin{array}{c}
N \\
m_{i}
\end{array}\right)}
$$

605

$$
p-\text { value }=\sum_{t_{i j}>T_{i j}}^{n_{j}} P\left(t_{i j}\right)
$$

606 where $N$ is the total number of reactions that associate with UniRef90 gene families and 607 pathways in MetaCyc, $m_{i}$ is the number of reactions within the $i$ th biomarkers, $n_{j}$ is the 608 total number of reactions within the $j$ th pathway, and $T_{i j}$ is the observed number of 609 reactions of pathway $j$ occurred in the biomarker $i$. This yielded a $p$-value for each 
610 biomarker and pathway. We selected the pathways with a $p$-value less than 0.05 as the

611 significantly enriched pathways of each biomarker.

612 Additionally, we used a widely used tool, LEfSe [11], to infer differentially abundance 613 pathways among three phenotypes. This was performed using pathway abundance 614 information of each sample from HUMAnN2.

616 Comparing IDAM with other methods

617 Biomarker inference of IDAM was compared with the other four top performing 618 unsupervised tools for grouping genes into functional modules [43], including biclustering619 based (QUBIC [24], ISA [25], and FABIA [26]) and decomposition-based (fastICA, an 620 improved method of ICA [23]). All tools were tested and compared based on the filtered 621 gene-sample matrix with 941,785 genes and 813 samples. We run these tools on the

622 Pitzer cluster of the Ohio Supercomputer Center with memory usage set to 300GB [44] 623 with default parameters. Specifically, QUBIC was implemented using the published source 624 code, while the other three were implemented by the R packages, isa2 0.3.5, fabia 2.36.0, 625 and fastICA 1.2.2, respectively.

627 Species-level analysis

628 For biomarkers from IDAM, ICA [23], QUBIC [24], and FABIA [26], we extracted species 629 that contribute to the abundance of genes within identified biomarkers, respectively, based 630 on HUMAnN2 output [28]. By this, community-level gene expression (abundance) was 631 decomposed into species-level data. The phylogenetic tree was generated using the 632 software GraPhIAn [45]. Since the biomarkers have been associated with phenotypes by 633 Z-test mentioned above, we assigned the species of each biomarker to the corresponding 
634 phenotype of the biomarker. For each phenotype, we counted the total number of species, 635 as well as the number that matched with the collected species. Then, the total number of 636 species in the phenotype dividing by the number of matched species was calculated as 637 the consistency index.

638 Besides, the tool, LEfSe [11], was used here for comparison. It is based on species 639 profiling from MetaPhIAn2 to identify statistically different species among the three 640 phenotypes $[46,47]$. The significantly differential species of each phenotype were aligned 641 to collected ones, by which we determined the number of matched species and calculated 642 the consistency of each phenotype.

643

644 Abbreviations

645 16S ribosome RNA: 16S rRNA;

646 Metagenomic: MG;

647 Metatranscriptomic: MT;

648 Inflammatory bowel disease: IBD;

649 Ulcerative colitis: UC;

650 Crohn's disease: CD.

651

652 Declarations

653 Ethics approval and consent to participate

654 Not applicable 


\section{Consent for publication}

656 Not applicable

\section{$657 \quad$ Availability of data and materials}

658 The datasets supporting the conclusions of this article are available in the IBDMDB

659 database (https://ibdmdb.org).

\section{Competing interests}

661 The authors declare that they have no competing interests.

\section{Funding}

663 This work was supported by National Key R\&D Program of China (2020YFA0712400), 664 National Nature Science Foundation of China (NSFC, 61772313 and 11931008), and 665 Interdisciplinary Science Innovation Group Project of Shandong University (2019).

\section{Authors' contributions}

667 J.Z. and B.L. conceived the basic idea. Z.L. carried out the computational analysis and 668 data interpretation. L.Z. and Q.W. designed and drew the figures. D.C. and Q.M. helped 669 polished the manuscript and all the authors wrote the manuscript. All authors read and 670 approved the final manuscript.

\section{Acknowledgements}

672 The authors would like to thank Anjun Ma for his assistance in the design of Fig. 1 and 673 Fig. 2.

674

675 References 
1. Sepich-Poore GD, Zitvogel L, Straussman R, Hasty J, Wargo JA, Knight R. The microbiome and human cancer. Science. 2021; 371: eabc4552.

2. Gilbert JA, Blaser MJ, Caporaso JG, Jansson JK, Lynch SV, Knight R. Current understanding of the human microbiome. Nature medicine. 2018; 24:392-400.

3. Consortium THMP. A framework for human microbiome research. Nature. 2012; 486:215-221.

4. Proctor LM, Creasy HH, Fettweis JM, Lloyd-Price J, Mahurkar A, Zhou W, Buck GA, Snyder MP, Strauss JF, Weinstock GM, et al. The Integrative Human Microbiome Project. Nature. 2019; 569:641-648.

5. Qin J, Li R, Raes J, Arumugam M, Burgdorf KS, Manichanh C, Nielsen T, Pons $\mathrm{N}$, Levenez $\mathrm{F}$, Yamada $\mathrm{T}$, et al. A human gut microbial gene catalogue established by metagenomic sequencing. Nature. 2010; 464:59-65.

6. Turnbaugh PJ, Ley RE, Mahowald MA, Magrini V, Mardis ER, Gordon JI. An obesity-associated gut microbiome with increased capacity for energy harvest. Nature. 2006; 444:1027-1031.

7. Dubinsky M, Braun J. Diagnostic and Prognostic Microbial Biomarkers in Inflammatory Bowel Diseases. Gastroenterology. 2015; 149:1265-1274.

8. Lecuit M, Lortholary O. Immunoproliferative small intestinal disease associated with Campylobacter jejuni. Med Mal Infect. 2005; 35:S56-58.

9. Paulson JN, Stine OC, Bravo HC, Pop M. Differential abundance analysis for microbial marker-gene surveys. Nat Methods. 2013; 10:1200-1202.

10. Liu Z, Ma A, Mathé E, Merling M, Ma Q, Liu B. Network analyses in microbiome based on high-throughput multi-omics data. Briefings in Bioinformatics. 2020; 22:1639-1655.

11. Segata N, Izard J, Waldron L, Gevers D, Miropolsky L, Garrett WS, Huttenhower C. Metagenomic biomarker discovery and explanation. Genome Biology. 2011; 12:R60.

12. Zhu Q, Jiang X, Zhu Q, Pan M, He T. Graph Embedding Deep Learning Guides Microbial Biomarkers' Identification. Frontiers in Genetics. 2019; 10:1182.

13. Wu Y, Jiao N, Zhu R, Zhang Y, Wu D, Wang A-J, Fang S, Tao L, Li Y, Cheng S, et al. Identification of microbial markers across populations in early detection of colorectal cancer. Nature Communications. 2021; 12:3063.

14. Snipen L, Angell I-L, Rognes T, Rudi K. Reduced metagenome sequencing for strain-resolution taxonomic profiles. Microbiome. 2021; 9:79.

15. Zhu Z, Ren J, Michail S, Sun F. MicroPro: using metagenomic unmapped reads to provide insights into human microbiota and disease associations. Genome Biology. 2019; 20:154.

16. Almeida A, Nayfach S, Boland M, Strozzi F, Beracochea M, Shi ZJ, Pollard KS, Sakharova E, Parks DH, Hugenholtz P, et al. A unified catalog of 204,938 reference genomes from the human gut microbiome. Nature Biotechnology. 2021; 39:105-114.

17. Franzosa EA, Morgan XC, Segata N, Waldron L, Reyes J, Earl AM, Giannoukos G, Boylan MR, Ciulla D, Gevers D, et al. Relating the metatranscriptome and metagenome of the human gut. Proceedings of the National Academy of Sciences. 2014; 111 :E2329-E2338.

18. Kasmanas JC, Bartholomäus A, Corrêa FB, Tal T, Jehmlich N, Herberth G, von Bergen M, Stadler PF, Carvalho André Carlos Ponce de Leon Ferreira d, Nunes da Rocha U. HumanMetagenomeDB: a public repository of curated and standardized metadata for human metagenomes. Nucleic Acids Research. 2020; 49:D743-D750. 
762

763

764

765

766

767

768

769

770

771

772

773

774

775

776

19. Yang J, Qin Y, Zhang T, Wang F, Peng L, Zhu L, Yuan D, Gao P, Zhuang J, Zhang $Z$, et al. Identification of human age-associated gene co-expressions in functional modules using liquid association. Oncotarget. 2017; 9:1063-1074.

20. Lathe III WC, Snel B, Bork P. Gene context conservation of a higher order than operons. Trends in biochemical sciences. 2000; 25:474-479.

21. Che D, Li G, Mao F, Wu H, Xu Y. Detecting uber-operons in prokaryotic genomes. Nucleic acids research. 2006; 34:2418-2427.

22. Lloyd-Price J, Arze C, Ananthakrishnan AN, Schirmer M, Avila-Pacheco J, Poon TW, Andrews E, Ajami NJ, Bonham KS, Brislawn CJ, et al. Multi-omics of the gut microbial ecosystem in inflammatory bowel diseases. Nature. 2019; 569:655662.

23. Hyvärinen A, Oja E. Independent component analysis: algorithms and applications. Neural Networks. 2000; 13:411-430.

24. Li G, Ma Q, Tang H, Paterson AH, Xu Y. QUBIC: a qualitative biclustering algorithm for analyses of gene expression data. Nucleic Acids Research. 2009; 37:e101-e101.

25. Bergmann S, Ihmels J, Barkai N. Iterative signature algorithm for the analysis of large-scale gene expression data. Phys Rev E Stat Nonlin Soft Matter Phys. 2003; 67:031902.

26. Hochreiter S, Bodenhofer U, Heusel M, Mayr A, Mitterecker A, Kasim A, Khamiakova T, Van Sanden S, Lin D, Talloen W, et al. FABIA: factor analysis for bicluster acquisition. Bioinformatics. 2010; 26:1520-1527.

27. Xie J, Ma A, Zhang Y, Liu B, Cao S, Wang C, Xu J, Zhang C, Ma Q. QUBIC2: a novel and robust biclustering algorithm for analyses and interpretation of largescale RNA-Seq data. Bioinformatics. 2020; 36:1143-1149.

28. Franzosa EA, Mclver LJ, Rahnavard G, Thompson LR, Schirmer M, Weingart G, Lipson KS, Knight R, Caporaso JG, Segata N, Huttenhower C. Species-level functional profiling of metagenomes and metatranscriptomes. Nature Methods. 2018; 15:962-968.

29. Morgan XC, Tickle TL, Sokol H, Gevers D, Devaney KL, Ward DV, Reyes JA, Shah SA, LeLeiko N, Snapper SB, et al. Dysfunction of the intestinal microbiome in inflammatory bowel disease and treatment. Genome Biology. 2012; 13:R79.

30. Imhann F, Vich Vila A, Bonder MJ, Fu J, Gevers D, Visschedijk MC, Spekhorst LM, Alberts R, Franke L, van Dullemen HM, et al. Interplay of host genetics and gut microbiota underlying the onset and clinical presentation of inflammatory bowel disease. Gut. 2018; 67:108-119.

31. Klaassen MAY, Imhann F, Collij V, Fu J, Wijmenga C, Zhernakova A, Dijkstra G, Festen EAM, Gacesa R, Vich Vila A, Weersma RK. Anti-inflammatory Gut Microbial Pathways Are Decreased During Crohn's Disease Exacerbations. Journal of Crohn's and Colitis. 2019; 13:1439-1449.

32. Schirmer M, Garner A, Vlamakis H, Xavier RJ. Microbial genes and pathways in inflammatory bowel disease. Nature reviews Microbiology. 2019; 17:497-511.

33. Franzosa EA, Sirota-Madi A, Avila-Pacheco J, Fornelos N, Haiser HJ, Reinker S, Vatanen T, Hall AB, Mallick H, Mclver LJ, et al. Gut microbiome structure and metabolic activity in inflammatory bowel disease. Nature microbiology. 2019; 4:293-305.

34. Kuballa A, Geraci M, Johnston M, Sorrentino D. The Gut Microbial Profile of Preclinical Crohn's Disease Is Similar to That of Healthy Controls. Inflamm Bowel Dis. 2020; 26:1682-1690.

35. Vich Vila A, Imhann F, Collij V, Jankipersadsing SA, Gurry T, Mujagic Z, Kurilshikov A, Bonder MJ, Jiang X, Tigchelaar EF, et al. Gut microbiota 
composition and functional changes in inflammatory bowel disease and irritable bowel syndrome. Sci Transl Med. 2018; 10:eaap8914.

36. Skoufos G, Kardaras FS, Alexiou A, Kavakiotis I, Lambropoulou A, Kotsira V, Tastsoglou S, Hatzigeorgiou Artemis G. Peryton: a manual collection of experimentally supported microbe-disease associations. Nucleic Acids Research. 2020; 49:D1328-D1333.

37. Wu J, Wei Z, Cheng P, Qian C, Xu F, Yang Y, Wang A, Chen W, Sun Z, Lu Y. Rhein modulates host purine metabolism in intestine through gut microbiota and ameliorates experimental colitis. Theranostics. 2020; 10:10665-10679.

38. Suzek BE, Huang H, McGarvey P, Mazumder R, Wu CH. UniRef: comprehensive and non-redundant UniProt reference clusters. Bioinformatics. 2007; 23:12821288.

39. Altschul SF, Gish W, Miller W, Myers EW, Lipman DJ. Basic local alignment search tool. J Mol Biol. 1990; 215:403-410.

40. Wang Z, Li G, Robinson RW, Huang X. UniBic: Sequential row-based biclustering algorithm for analysis of gene expression data. Sci Rep. 2016; 6:23466.

41. Ben-Dor A, Chor B, Karp R, Yakhini Z. Discovering local structure in gene expression data: the order-preserving submatrix problem. J Comput Biol. 2003; 10:373-384.

42. Caspi R, Billington R, Keseler IM, Kothari A, Krummenacker M, Midford PE, Ong WK, Paley S, Subhraveti P, Karp PD. The MetaCyc database of metabolic pathways and enzymes - a 2019 update. Nucleic Acids Res. 2020; 48:D445d453.

43. Saelens W, Cannoodt R, Saeys Y. A comprehensive evaluation of module detection methods for gene expression data. Nature Communications. 2018; 9:1090.

44. Center OS. Ohio Supercomputer Center. 1987.

45. Asnicar F, Weingart G, Tickle TL, Huttenhower C, Segata N. Compact graphical representation of phylogenetic data and metadata with GraPhIAn. PeerJ. 2015; 3:e1029.

46. Segata N, Waldron L, Ballarini A, Narasimhan V, Jousson O, Huttenhower C. Metagenomic microbial community profiling using unique clade-specific marker genes. Nature Methods. 2012; 9:811-814.

47. Truong DT, Franzosa EA, Tickle TL, Scholz M, Weingart G, Pasolli E, Tett A, Huttenhower C, Segata N. MetaPhlAn2 for enhanced metagenomic taxonomic profiling. Nature Methods. 2015; 12:902-903.

\section{Supplementary Information}

\section{Additional file 1: Supplementary material}

This supplementary material includes details in the Problem formulation and Methods part.

Section 1 illustrates why the problem we formulated is NP-hard. Sections 2 and 3 describe how to do discretization and select parameter $w$. 


\section{Additional file 2: Supplementary results}

822 This file includes supplementary results in the Result part and parameter selection of $\mathrm{w}$ in 823 the Method part. The first is an overview of Additional file 2. Table S1 shows the size of 824 identified gene modules from IDAM. Table S2 shows the Z-score and $p$-value of each 825 module for three phenotypes. Table S3 shows the adjusted $p$-value from Wilcoxon signed826 rank test. Table S4 shows pathways enriched for identified biomarkers from IDAM. Table 827 S5 shows the species associated with different phenotypes from IDAM. Table S6 shows 828 the species associated with different phenotypes collected from previous studies. Table 829 S7 shows the performance of IDAM with different parameter $w$, which is attached to 830 Methods and Additional file 1: Section 3. 
a

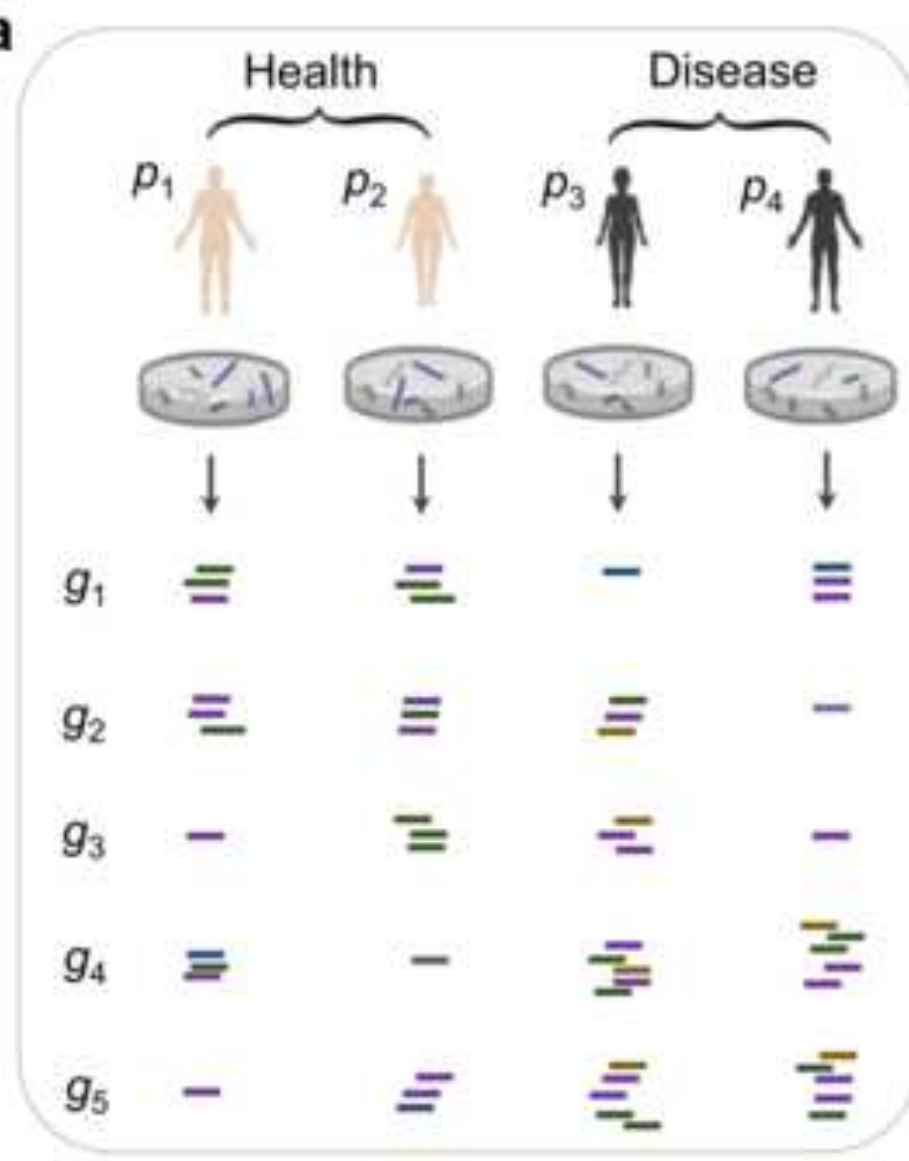

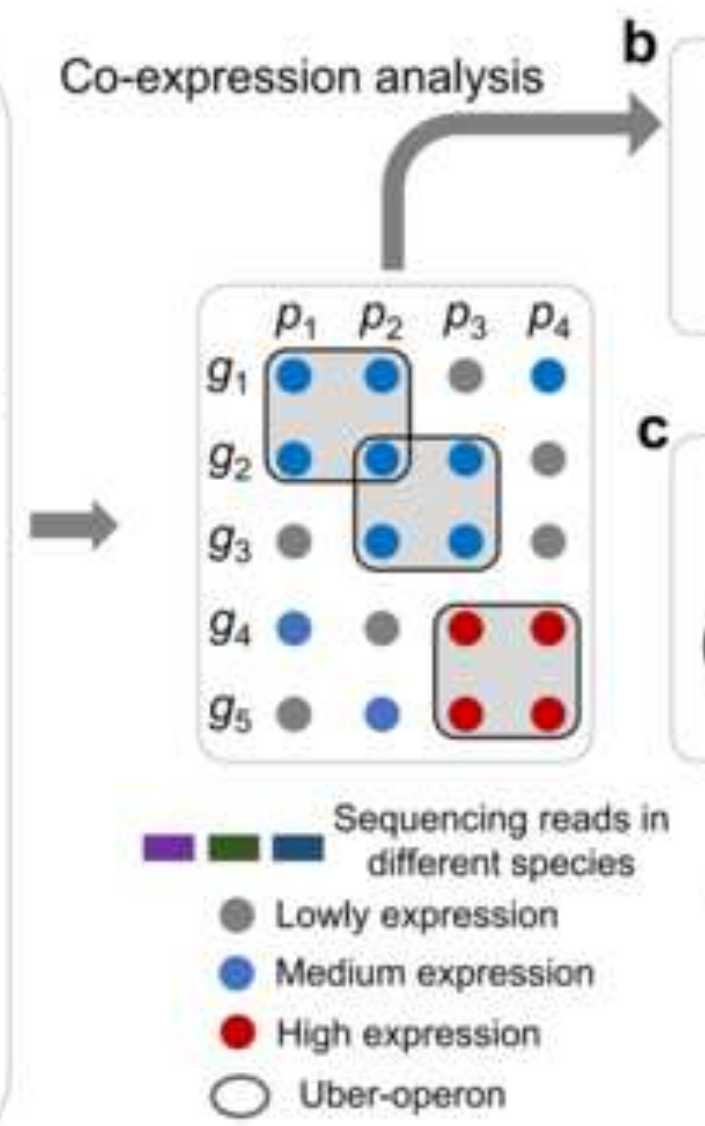

b Co-expressed gene modules

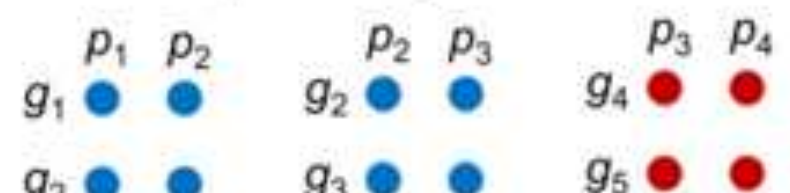

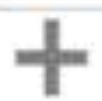

Uber-operon inclusion

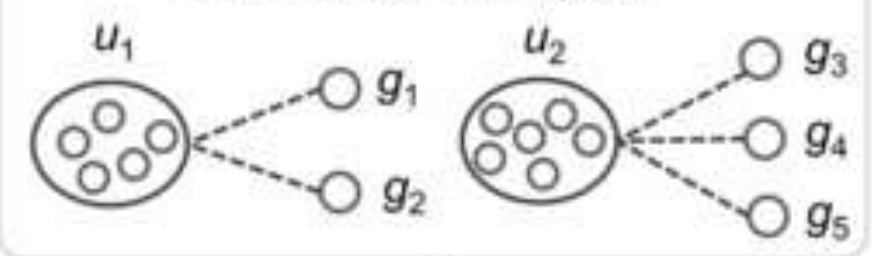

Functionally related gene modules

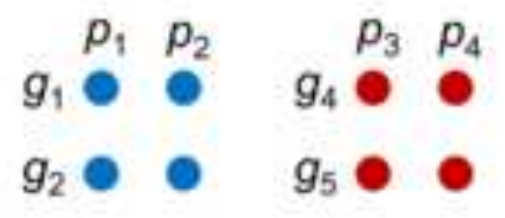


a

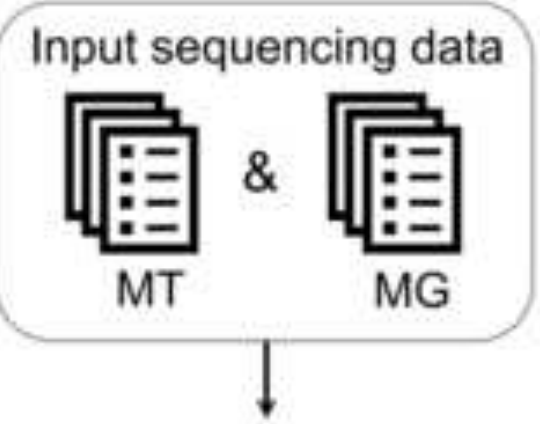

Matrix construction

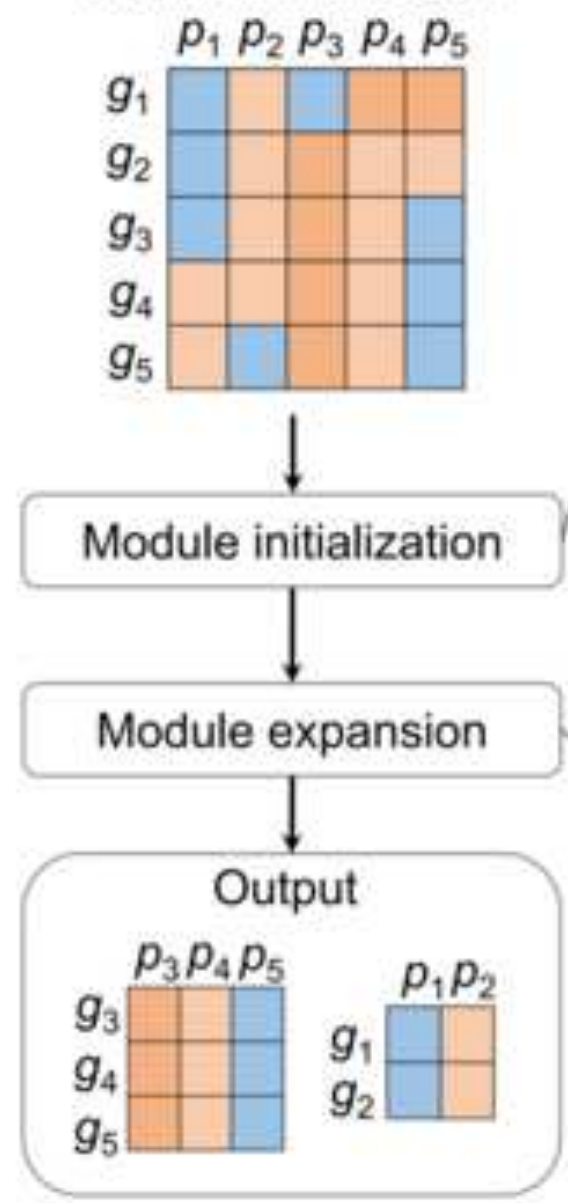

b
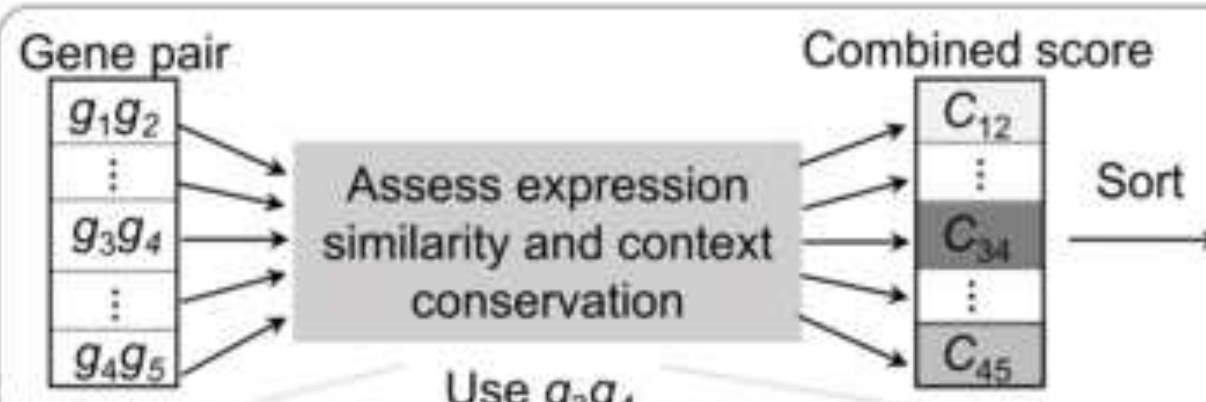

Use $g_{3} g_{4}$

as an example

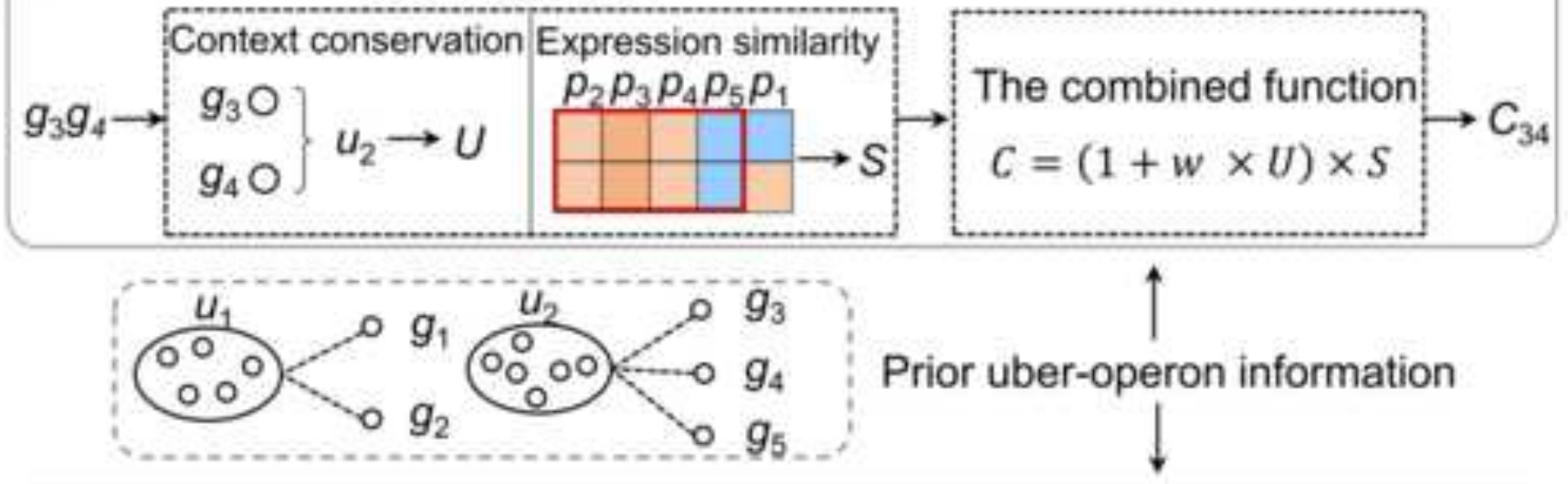

C
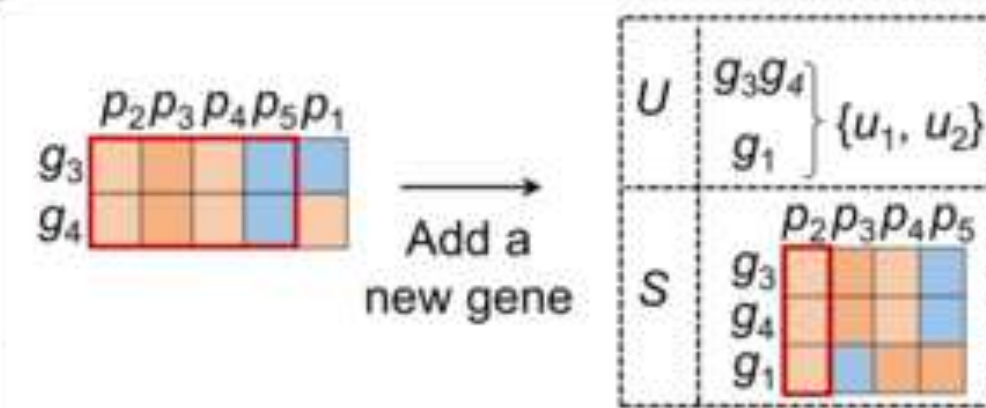

$g$
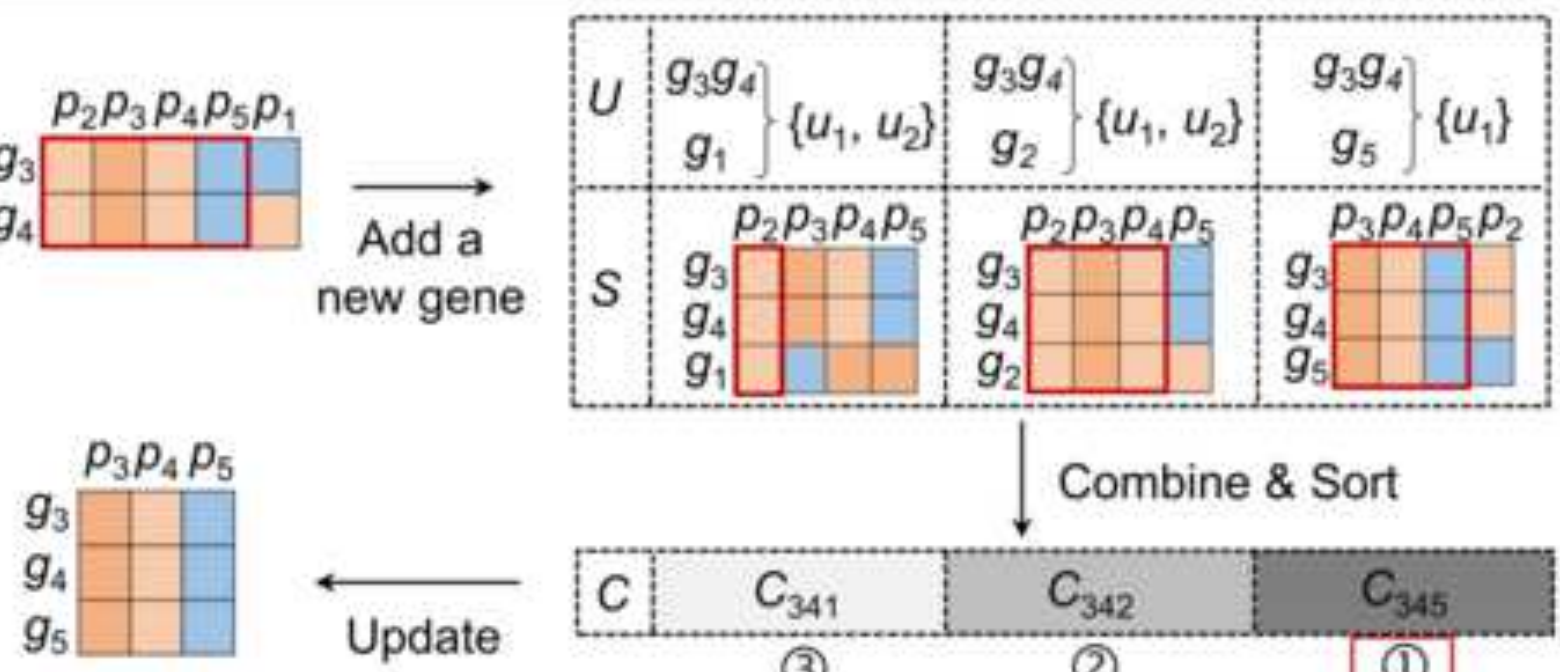
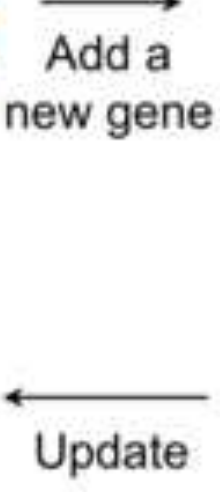
new gene

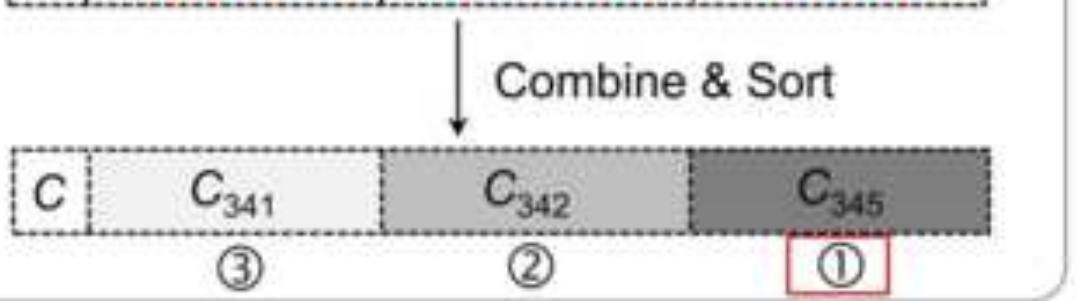



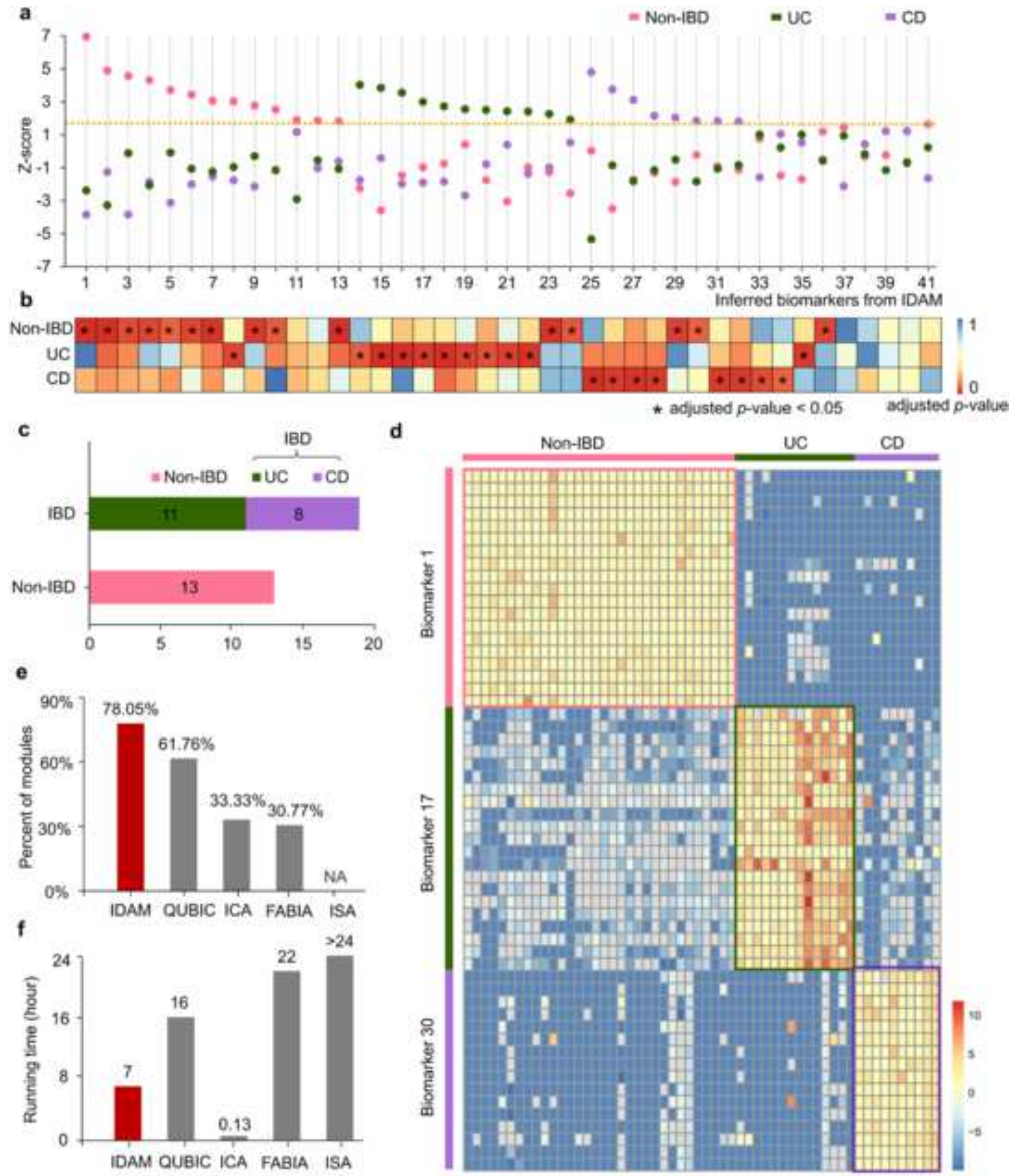

$\log _{3}($ expression) 
a

b

\begin{tabular}{cccl}
\hline Method & Non-IBD (34) & UC (32) & \multicolumn{1}{c}{ CD $(90)$} \\
\hline IDAM & $17 / 37(45.95 \%)$ & $\mathbf{6 / 6 4}(\mathbf{9 . 3 8} \%)$ & $\mathbf{9 / 1 3}(69.23 \%)$ \\
QUBIC & $12 / 36(33.33 \%)$ & $2 / 35(5.71 \%)$ & $10 / 49(20.41 \%)$ \\
ICA & $18 / 95(18.95 \%)$ & $6 / 40(15.00 \%)$ & $7 / 37(18.92 \%)$ \\
FABIA & 0 & $7 / 151(4.64 \%)$ & $11 / 115(9.57 \%)$ \\
LEfSe & $11 / 37(29.73 \%)$ & $2 / 37(5.41 \%)$ & $4 / 20(20 \%)$ \\
\hline
\end{tabular}

Escherichia coli •

Eubacterium rectale $\circ$ Lachnospiraceae bacterium $。$ 51 63FAA

Enterocloster citroniae Enterocloster bolteae $\bullet$ Enterocloster clostridioformis 0 Roseburia hominis • Roseburia intestinalis • Blautia obeum • Blautia producta $\bullet$ Ruminococcus lactaris o Clostridium symbiosum • Anaerostipes hadrus • Eubacterium siraeum 0 Oscillibacter sp KLE $1728^{\circ}$

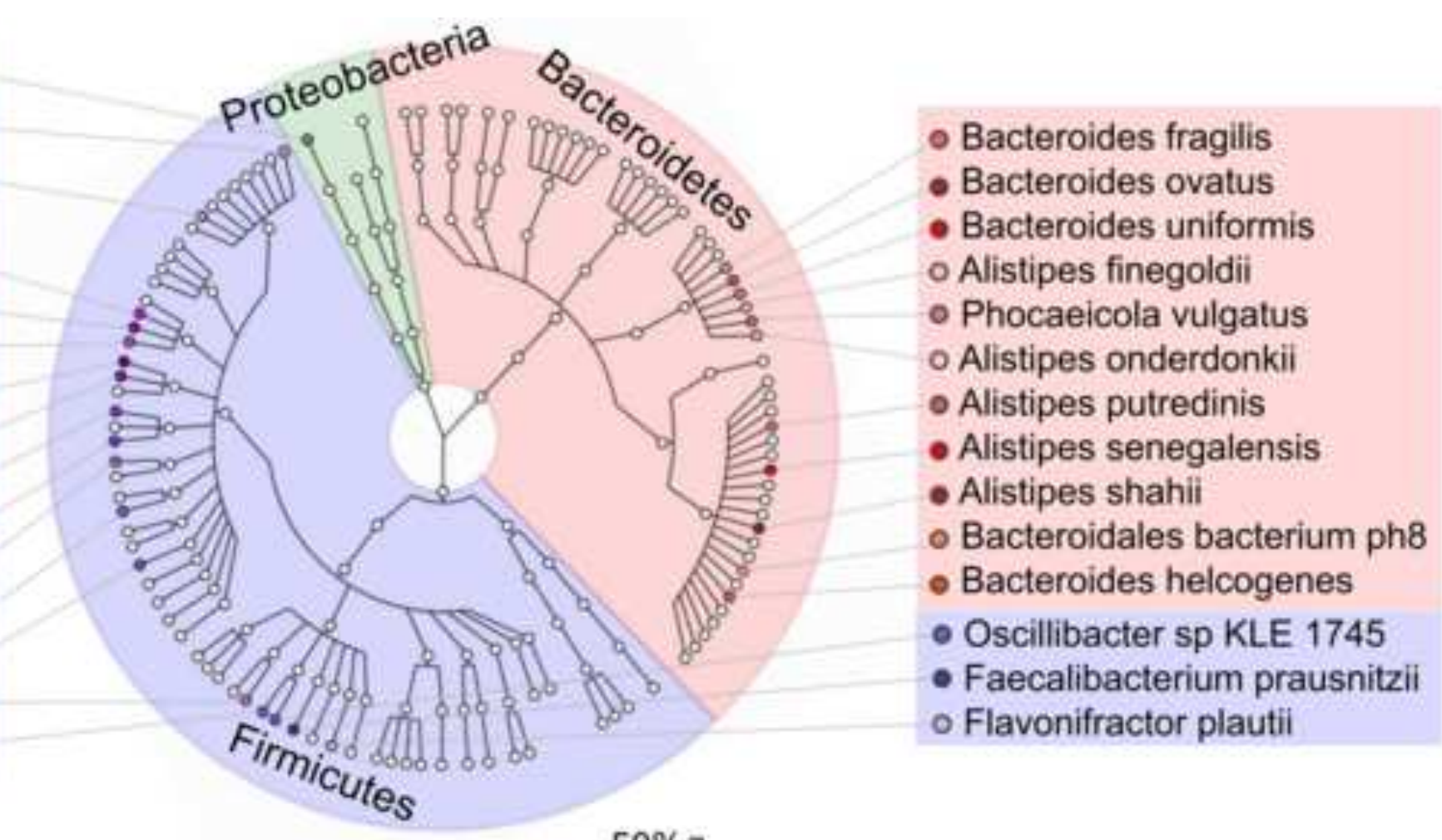

c $50 \%]$

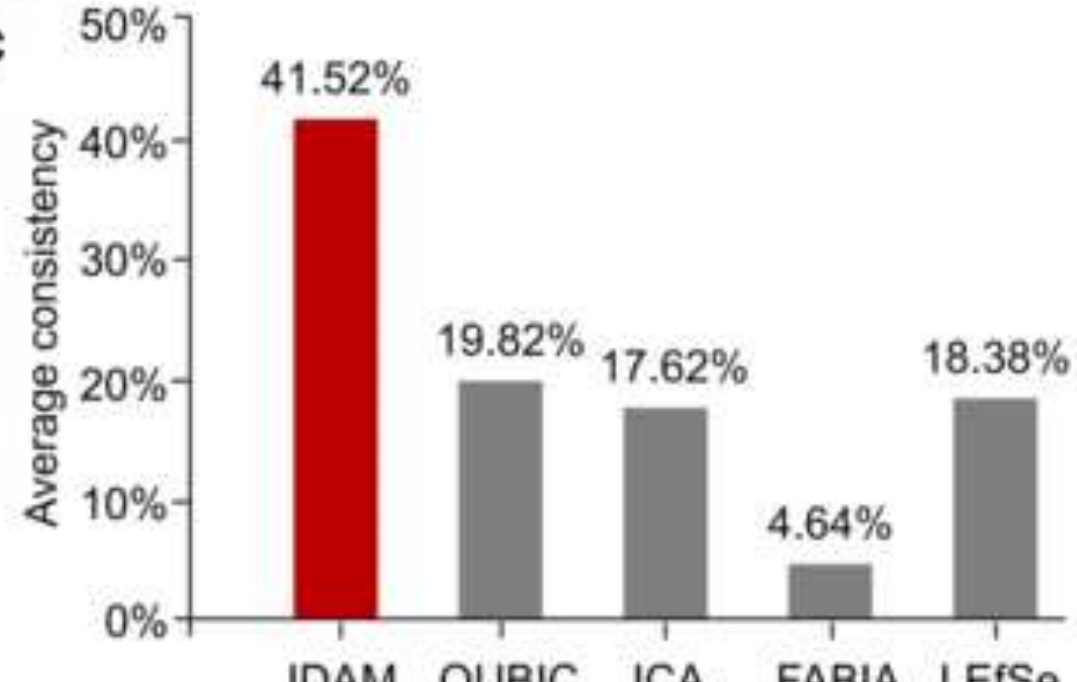




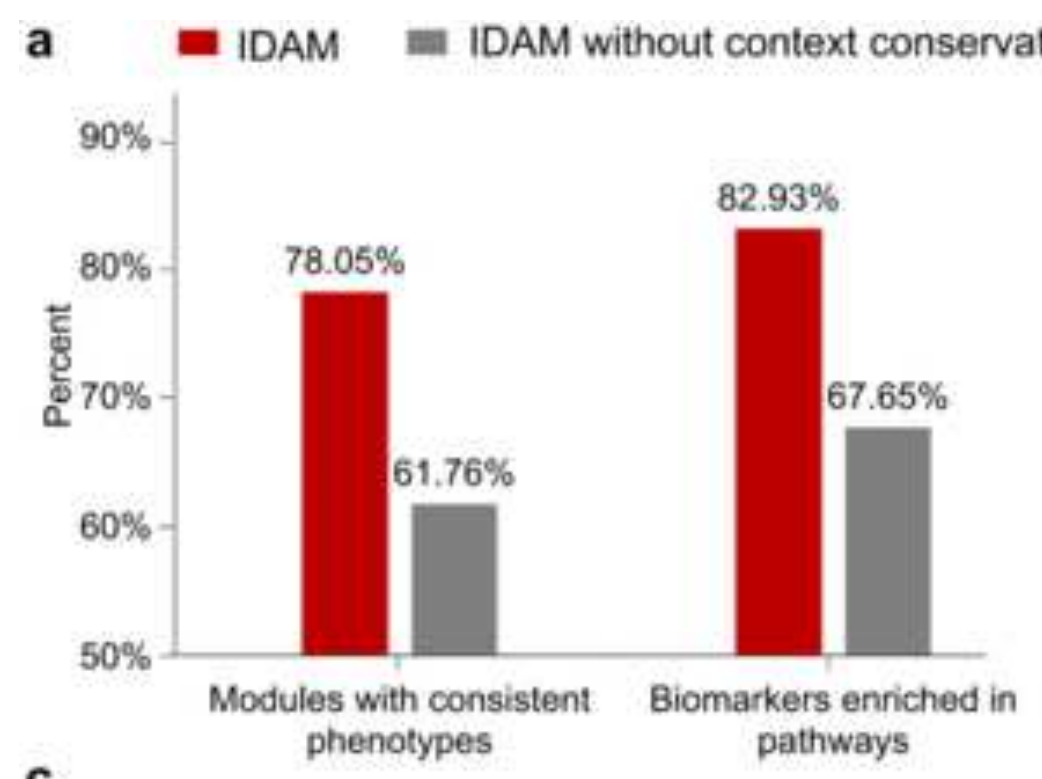

c

3.35

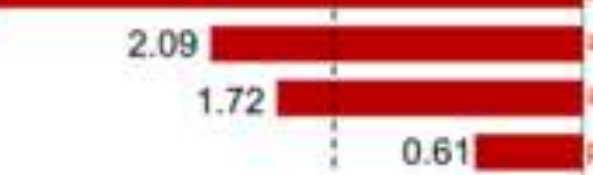

MeO biasynthesis

aderine and adencsine salvage iti adenosine nucleotides degradation il superpathway of (Kdo), fipid A biosynthesis peptidoghycan biosynthesis NV Ipid IVA biosynthesis lactose and galactose degradation ! 0.14

N-acetyl-D-galactosamine degradation D-gatactosamine and N-acetyl-D. galactosamine degradation

\section{.}

0.44

0.47 0.59 0.76 galactitol degradation 1,0 $\log _{10}(p$-value $)$

\section{b}

= Non-IBD

- UC

$=\mathrm{CD}$
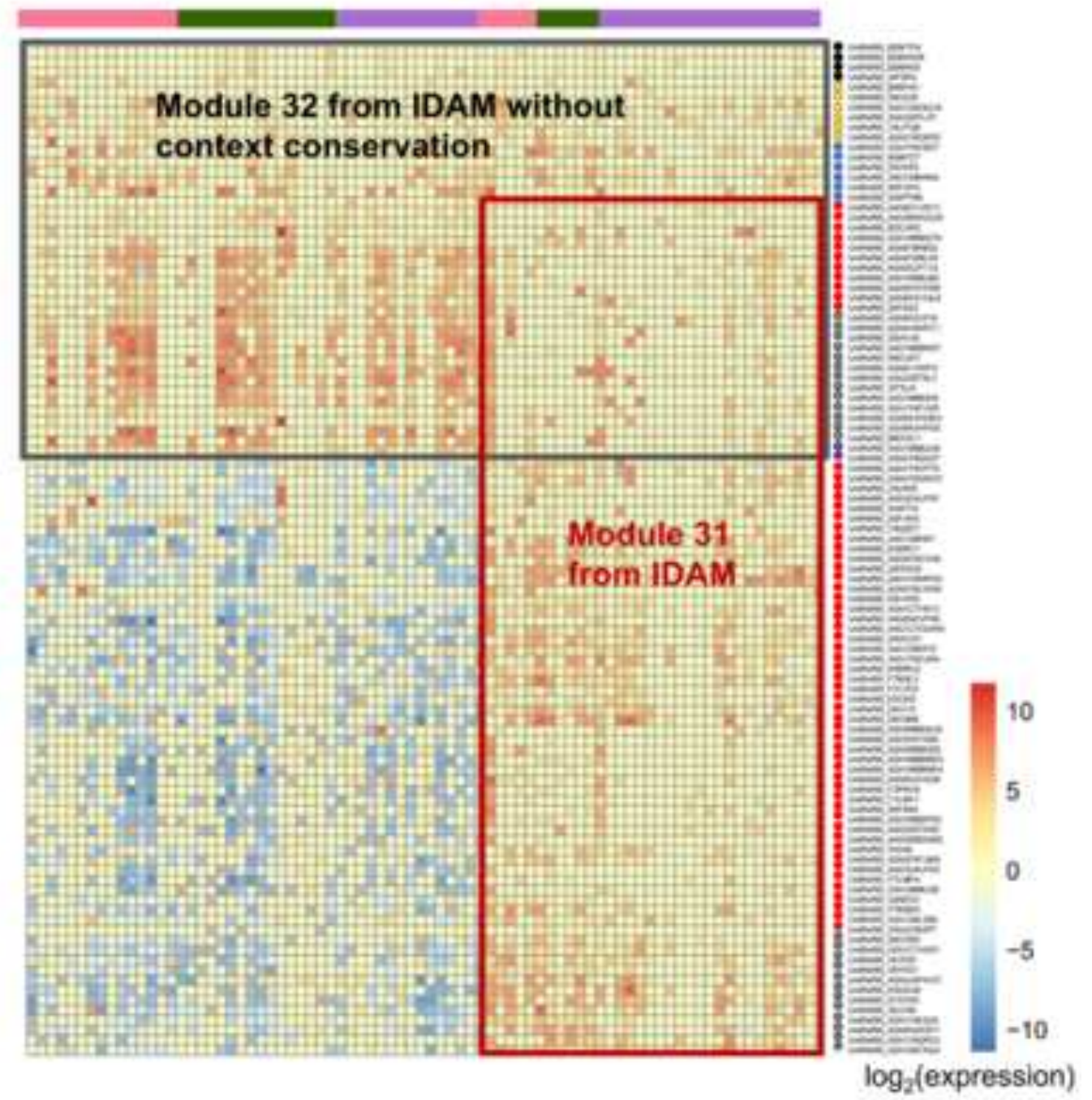
SupplementaibRAWatejeht doi: https://doi.org/10.1101/2021.09.13.460160; this version posted October 21, 2021. The copyright holder for this preprint (which was not certified by peer review) is the author/funder. All rights reserved. No reuse allowed without permission.

\section{Click here to access/download Supplementary Material Additional file 1.docx}

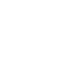


Supplementaib/R (which was not certified by peer review) is the author/funder. All rights reserved. No reuse allowed without permission.

\section{Click here to access/download \\ Supplementary Material Additional file 2.xlsx} Additional file 2.xisx

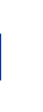

(n)

(6) 